\title{
Spreading of molecularly thin wetting films on solid interfaces
}

\author{
S.F.Burlatsky ${ }^{1}$, A.M.Cazabat ${ }^{2}$, M.Moreau ${ }^{3}$, G.Oshanin ${ }^{4}$, S.Villette ${ }^{2}$ \\ ${ }^{1}$ Department of Chemistry BG - 10, University of Washington, Seattle, WA 98195 USA; \\ sfburlat@ringa.chem.washington.edu \\ ${ }^{2}$ Laboratoire de Physique de la Matière Condensée ${ }^{\diamond}$, Collège de France, 11 Place Marcelin \\ Berthelot, 75231 Paris Cedex 05, France \\ ${ }^{3}$ Laboratoire de Physique Théorique des Liquides ${ }^{\S}$ Université Paris VI, 4 Place Jussieu, 75252 \\ Paris Cedex 05, France \\ ${ }^{3}$ Centre de Recherche en Modélisation Moléculaire, Service de Physique Statistique et Probabilités, \\ Université de Mons-Hainaut, 20 Place du Parc, 7000 Mons, Belgium; gleb@gibbs.umh.ac.be
}

\begin{abstract}
.
In this paper we study kinetics of spreading of thin liquid films on solid interfaces. We present an overview of current experimental picture and discuss available theoretical approaches and their limitations. We report some new experimental results on spreading of molecularly thin liquid films and propose an analytically solvable microscopic model, which reproduces experimentally observed behaviors and provides a seemingly plausible explanation of the underlying physical processes.
\end{abstract}




\section{INTRODUCTION}

The spreading of liquid droplets on solid interfaces is important in many technological and natural processes, such as lubrication, painting, glueing, coating, emulsion, dyeing and oil recovery from porous rocks. In all instances, a precise knowledge of conditions and laws of spreading is required for efficient practical applications. Systematic experimental studies provide now a great wealth of information revealing rich behavior dependent on the structural details and interactions in the co-existing phases. However, currently available theoretical developments don't explain all pertinent features of this complex phenomenon, substantiated by experiments. Particularly, still little is known about physical mechanisms which cause growth of molecularly thin films arising in the process of spreading of macroscopically large liquid droplets.

In this paper we focus on the particular issue of thin liquid films spreading; we discuss here results of earlier experimental studies, as well as report some new ones, which substantiate remarkable universal (i.e. independent of the liquid/solid system in question) behavior of such films and also propose an analytical description, which reproduces experimentally observed growth laws and sheds the light on the underlying physical processes.

The paper is outlined as follows: Section II contains a brief overview of current experimental and theoretical pictures of wetting films spreading. In section III we report results of recent ellipsometric experiments on spreading of non-volatile liquid droplets, which produce molecularly thin films. We present the data on the time evolution of thickness profiles of spreading droplets and also on the growth rate of the monolayer on top of solid substrate. In Section IV we formulate our analytical model, write down basic equations and discuss their solutions for both the case of spreading of circular liquid droplets on solid interfaces and growth of monolayers on vertical solid wall immersed in liquid bath (capillary rise geometries). Finally, in Section V we conclude with a summary of our results and discussion. 


\section{AN OVERVIEW OF EXPERIMENTAL AND ANALYTICAL RESULTS}

When a liquid droplet is placed on a flat solid substrate, three different interfaces come into play and three interfacial tensions are involved; respectively, the tensions of the solidliquid, solid-vapour and liquid-vapour interfaces. The qualitative behavior of the droplet is merely controlled by the sign of $S$ - the so-called spreading parameter, which equals the free energy difference between a bare solid and a solid covered by a thick liquid layer. When

$S>0$ the droplet spreads spontaneously and tends to shield the solid surface. Such a situation is called the complete wetting. The case where $S$ is negative is referred to as a partial wetting. Here the liquid remains in the form of a droplet; it may contract or dilate but ultimately comes to an equilibrium bead-like shape and the liquid droplet ceases to move.

The spreading of a pure, nonvolatile liquid droplet on a smooth, homogeneous substrate is now well understood at the macroscopic scales $[1$ - 8]. Continuum hydrodynamic descriptions provide general laws for the time evolution of such macroscopic properties as the radius $R_{m l e}$ of the macroscopic liquid edge, the height of the droplet, its shape and the contact angle. These results suggest that in the complete wetting case the time evolution of the macroscopic properties is rather insensitive to the specific details of the liquid/solid system in question and follows universal time dependences. In particular, $R_{\text {mle }}$ was found to grow in proportion to $t^{1 / 10}, t$ being time, in case of sufficiently small drops such that the gravity effects are negligible; and a slightly stronger dependence, $R_{m l e} \sim t^{1 / 8}$, has been predicted and verified experimentally for situations in which gravity is important.

The most recent progress in the complete wetting case resulted from experimental works [9 11] which examined kinetics of spreading on the mesoscopic and microscopic scales. The salient feature here is the appearance of a thin film, commonly referred to as the "precursor", which extracts from the droplet and advances ahead of the macroscopic liquid edge. The thickness of the film may vary considerably depending on the particular liquid/solid system and may range from several (molecular size) to hundreds of angstroms; its linear extension 
is macroscopically large (in the range of millimeters) at sufficiently large times.

Extensive ellipsometric studies [9]1] (see also recent works [12 15] and pertinent references therein) have thoroughly examined the growth of the precursor film and reached a rather surprising conclusion: as long as the droplet plays a role of a reservoir for the film, the radius of the film $R(t)$ obeys a universal law $R(t) \sim \sqrt{t}$ regardless of the nature of the species involved. To be more specific, the same $\sqrt{t}$-law for growth of the precursor film shows up in experiments involving droplets of different types of simple liquids, polymer or surfactant melts, and carried out on different types of solid substrates (either bare or grafted). Furthermore, it is observed also in the capillary rise geometries [16, 17, in which a vertical solid wall is put in contact with a bath of liquid. In such experimental situation a film of microscopic thickness extracts from the macroscopic meniscus and creeps upwards along the wall; the front of the film being planar compared to the radially-symmetric one observed for circular droplets deposited on a horizontal substrate. The linear extension of the film again was found to grow in proportion to $\sqrt{t}$ within a rather extended time domain, until at very large times (which, in fact, may be several years) and at large altitudes above the macroscopic meniscus the growth is truncated due to gravity. Finally, for several substrates an even more remarkable behavior of spreading droplets was observed: experiments have shown that several molecularly thin films may advance together, stacked on top of one another and thus forming a stepped, "terraced" shape of the drop [9 11] (see, e.g. Fig.1 of the present paper). Also in this "terraced" wetting case, at sufficiently short times the radii of different monolayers were found [9 11, 14, 15] to spread out as $\sqrt{t}$, with the prefactor being a decreasing function of the distance from the substrate; the bottom layer moves outwards fastest, followed by the second from the bottom and etc.

Computer simulations are now able to reproduce the experimentally observed behavior employing different types of simulation techniques. In particular, MD simulations with Lennard-Jones chain-like molecules, performed in [18 20], exposed the spreading in the form of distinct layers with their radii growing as $\sqrt{t}$. In the simulations in [21,22] an Ising-type lattice-gas model with Kawasaki dynamics has been employed, which has also yielded the 
$\sqrt{t}$-law for growth of the precursor film. Details of these and earlier numerical approaches were recently reviewed in [23].

Meanwhile several analytical approaches are available, which aim on the explanation of the $\sqrt{t}$-law and of the "terraced" wetting phenomenon:

Joanny and de Gennes [24] have developed a continuum hydrodynamic theory and found that the $\sqrt{t}$-law originates from a diffusive-type molecular motion with inhomogeneous diffusion coefficient dependent on the local disjoining pressure. This theory presumes, however, that the film thickness remains at least in the mesoscopic range, where the continuum hydrodynamics description is still appropriate. Thus this approach does not explain the growth of monolayers. Thin liquid films on top of solid interfaces can not be viewed as a true liquid phase. In such liquid/solid systems the disordered liquid state contends with the ordering potential of the solid, which spans the liquid layer resulting in a markedly different behavior compared to these occuring in the bulk liquids. For instance, experiments reveal intriguing effects of solid or glassy-like response to an external shear or anomalously high relaxation times [25 28]. Cazabat et al. [17] proposed a phenomenological extension of the hydrodynamic approach [24] for the description of molecularly thin films spreading; in this description basic equations of the theory by Joanny and de Gennes [24] have been adopted, but a different origin of frictional forces has been assumed to account for the molecular behavior.

Further on, a qualitatively different semicontinuum model, proposed by de Gennes and Cazabat [29], treated the drop as a layered structure, in which each layer is an incompressible, two-dimensional fluid. In this picture the motion of the fluid molecules in the direction perpendicular to the layers is allowed and the latter may grow by the accretion of molecules at their edges from the layers above and below. Fluid particles experience an attractive force from the substrate what makes the lower layers energetically more favorable and causes spreading of the layers in the lower part of the drop. This model predicts correct time behavior of the advancing monolayers at long times, when one expects the difference between 
the radii of neighboring layers to be large. In this regime the radii of monolayers are found to scale with time as $\sqrt{t / \ln (t)}$. Assumption of incompressibility, however, renders inadequate description for short times, when the radii of monolayers are comparable. Besides, the validity of the macroscopic hydrodynamic description of dissipative forces, employed in the model by de Gennes and Cazabat [29], requires more extensive microscopic justification. Lastly, an interesting non-equilibrium statistical physics description of the precursor spreading was based on the solid-on-solid-model (SOSM) approximation [30. Abraham et al 31,32 have developed an interfacial model for the dynamics of a non-volatile fluid edge, in which the time evolution of the liquid-vapour interface was analysed in terms of Langevin dynamics for the displacement of horizontal solid-on-solid strings at increasing heights from the substrate. A free energy function associated with any configuration of the interface revealed a competition between surface tension and substrate interaction. The model allowed an analytical solution, which showed an extraction of a precursor film and also "terraced" forms of the dynamical thickness profiles. This approximation predicted, however, a constant velocity for the advancing precursor film, i.e. $R(t) \propto t$, what contradicts apparently to experimental observations, and shows thus that such an approach discards some important aspects of spreading phenomenon. To avoid this inconsistency De Coninck et al. [33] elaborated a different, "columnar" version of the SOSM, which takes into account entropic repulsion effects, but, nonetheless, obtained a precursor film extending linearly in time.

To summarize this section, we conclude that experimental studies evidence the universal behavior of spreading monolayers; wetting films are found to grow in proportion to $\sqrt{t}$, regardless of the nature of the liquid/solid system. The origin and the underlying physics of such a growth remain, however, incompletely understood; computer simulations are now able to reproduce it, but no convincing theoretical approaches have been, as yet, developed. 


\section{EXPERIMENTAL}

Experimental thickness profiles of spreading drops are conveniently studied using spatially resolved ellipsometry. Ellipsometry allows to measure the thickness of a film on a substrate by analyzing the change of polarization of an optical beam at reflection. For specific configurations, the optical beam can be focussed on the sample thus improving the lateral resolution.

The present study was performed with a polarization modulated ellipsometer working at a single wavelength (6328 $\AA$, He-Ne laser) and at Brewster angle. The substrates used were oxidized silicon wafers, either bare or bearing a grafted hydrophobic layer. The liquids involved were either light polymers (polydimethylsiloxanes, trimethy-terminated, abbreviated as PDMS) or nonvolatile silane derivative, like tetrakis(2-ethyl-hexoxy)silane, abbreviated as TK. Specifically, the PDMS is a chain-like molecule, whose molecular mass in our case is 9300 (it contains 126 monomers, polydispersity index being 1.09) and the transverse size is $7 \AA$. The TK molecule is spherical, with a diameter around $10 \AA$. The optical index of the silica, of the grafted layer if any, and of the liquids under study are close, around 1.4, while the index of the underlying silicon is around 3.8 for the red light. This high contrast between silicon and the layers on top of it ensures a good thickness resolution, $0.1 \AA$ per measurement for a measurement time $20 \mathrm{~ms}$.

The lateral resolution of the setup is $25 \mu \mathrm{m}$. At this scale, the substrate roughness (typically $5 \AA \mathrm{rms}$, with a characteristic length along the substrate $\approx 200 \AA$ ) is smoothed out and thus the thickness profiles are not noisy. Note that for the low thickness considered, an independent determination of the thickness and of the optical index is not possible: All the layers on top of silicon are seen as a whole and the corresponding optical path is converted into "thickness" by dividing by the average bulk index value 1.4 [34]. The response of the setup being linear in this range, the relative error on the thickness is the relative error on the index. The baseline on the profiles is the thickness of the silica layer.

Now, in experiments we have monitored the time evolution of two different properties 
- the thickness profiles of spreading droplets and the radius of the first layer on top of solid substrate. Results of experiments are plotted in Figs.1-4; two first figures concern the spreading of the TK droplets at different relative humidities, while Figs. 3 and 4 represent analogous data for the droplets composed of the PDMS molecules. Being interested mainly in the microscopic details of the thickness profiles, we have plotted the thickness profiles of spreading droplets (the $Y$-axis) in the range of angstroms, while the radial size of the droplet is presented on the millimeter scale, which causes a huge disproportion between the scales on the $X$ and the $Y$-axis. Consequently, the thickness profiles recorded at relatively short times after the deposition of the droplet on the substrate are out of scale.

In Fig. 1 we present the ellipsometric profiles of the TK droplets spreading on a bare silicon wafer at relative humidity $60 \%$. The profiles were recorded consequently at $30 \mathrm{mn}$, $5 \mathrm{~h} 45,22 \mathrm{~h}, 30 \mathrm{~h}, 4$ days, 5 days and, eventually, 11 days after the deposition of the drop. It is clearly seen here that with increasing times the profiles widen out and flatten, the volume of the drop being constant, forming well-defined terraces of the step thickness $\approx 10 \AA$, i.e. comparable to the molecular size. The baseline has been substracted, i.e. zero thickness corresponds to the surface of the silica.

In Fig. 2 we plot experimental thickness profiles (top) and the time dependence of the radius of the first layer (bottom) corresponding to the short time regime in spreading of a relatively large droplet of TK (at low relative humidity, $20 \%$ ). Profiles are measured at $1 \mathrm{~h}$, $2 \mathrm{~h} 15,4 \mathrm{~h}, 8 \mathrm{~h}$ and $20 \mathrm{~h} 15$ respectively after the deposition of the droplet. In contrast to the situation depicted in Fig.1, in this case the centre of the drop plays as a reservoir for the film during the whole period of time. We again stress the huge disproportion between the scales on the $X$ and the $Y$-axis, to avoid the impression that the thicknesses and the linear extensions of layers are comparable to each other. The baseline has not been substracted, i.e. the thickness of the silica layer is $\approx 19 \AA$. On bottom of the Fig. 2 we plot the radius $R(t)$ of the first layer versus the square root of the elapsed time. Straight line,

$$
R(t)=R_{m l e}+\sqrt{\alpha t},
$$


with $\alpha \approx 1.2 \times 10^{-10} \mathrm{~m}^{2} \mathrm{~s}^{-1}$ gives the fit of experimental data.

Fig.3 describes the thickness profiles and the time dependence of the precursor film radius in case of the PDMS droplet spreading on a grafted hydrophobic surface; the silica is now covered by a layer of trimethyl groups. The total thickness of silica and grafted layer is $\approx 23$

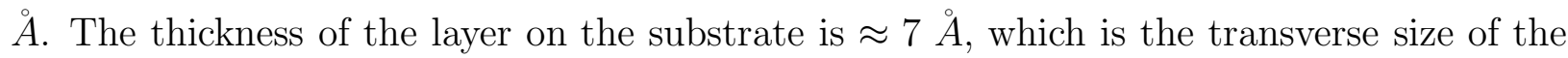
PDMS molecule; this means that the PDMS chains are lying flat on the top of the substrate. On bottom of Fig.3 we plot $R(t)$ versus the square root of time. The straight line gives the fit of experimental results by the function in Eq.(1), in which $\alpha \approx 5.5 \times 10^{-11} m^{2} s^{-1}$. The last experimental point is off the straight line, because the "reservoir" has disappeared to this moment. The radius will now stay constant for months.

In Fig.4 we present experimental thickness profiles obtained during spreading of the PDMS droplets in the conditions, in which the bare silica is exposed to high relative humidity $(\mathrm{RH}=90 \%)$ and therefore is covered by a thin adsorbed layer of water. Here, the thickness of water is $\approx 4 \AA$, i.e. of the order of the transverse size of the PDMS molecules. The total thickness of silica and water is $\approx 18 \stackrel{\AA}{A}$. In this case a single monolayer of a constant thickness shows up, advancing ahead of the macroscopic liquid edge. The macroscopic edge does not move significantly during the whole elapsed time. The curves on the top of Fig.4 correspond to the profiles of a large droplet at 1h30, 2h30 and 5h after deposition; the curves on the bottom of Fig.4 describe the thickness profiles of a small drop after 2h, $3 \mathrm{~h} 30$ and 5h15 (monolayer). The time dependence of the radius of the monolayer is fitted by Eq.(1) with $\alpha \approx 10^{-9} m^{2} s^{-1}$, which is substantially greater than that obtained in the previous case (Fig.3). The reason is that the friction between spreading molecules and the surface is here considerably decreased, without changing the interaction in a significant way [13]. 


\section{ANALYTICAL DESCRIPTION OF SPREADING OF MONOLAYERS ON SOLID INTERFACE.}

In this section we formulate a microscopic, analytically solvable model describing spreading of molecularly thin wetting films, write down basic equations and discuss their solutions for both the case of capillary rise geometries and circular droplets deposited on a horizontal substrate. Some aspects of this model and of the solutions have been discussed in [35, 36].

\section{A. The model}

The analytical model includes three basic points, which can be succinctly formulated as follows:

First, we suppose that the wetting film is in equilibrium with the bulk liquid (the macroscopic part of the droplet deposited on a horizontal substrate or the liquid bath in case of capillary rise geometries), which acts as a reservoir of particles and feeds the film. We stipulate that the "reservoir" has an infinite capacity and thus the bulk liquid maintains a constant density of fluid particles at the macroscopic liquid edge (see Fig.5).

Second, dynamics of fluid particles on top of solid interface is viewed as a symmetric, activated random hopping motion, constrained by hard-core interactions. In such a picture the wetting film can be thought off as being a hard-sphere fluid adsorbed on solid interface; the attractive interactions between the fluid particles are, as yet, discarded.

Third, we account for these cohesive interactions by introducing an impenetrable "liquidvapour" interface, which encloses the hard-sphere fluid and influences dynamics of fluid particles being in the vicinity of the interface. The physical properties of this "liquidvapour" interface are described in terms of the SOSM approximation 31,32; using some physical arguments we relate the surface tension of this interface to the overall cohesion energy of the particles in the film.

Now, let us describe the model more precisely: 
- Fluid density at the macroscopic liquid edge

We denote the density of fluid particles at the macroscopic liquid edge (MLE) (see Fig.5) as $\rho_{0}$. The value of $\rho_{0}$ can be estimated employing essentially the same type of reasonings as those used for the derivation of the Langmuir adsorption isotherm [37]: Suppose that one has a "vacancy" directly at the macroscopic liquid edge and a fluid particle in the bulk phase, being at the distance of a single "jump" from the MLE. Let us denote now as $E_{\downarrow}$ the energy gained by moving this particle onto the vacancy. Then, in the limit $\beta E_{\downarrow} \gg 1$, $\beta=1 / k T$, we find that $\rho_{0}$ is simply

$$
\rho_{0} \approx 1-\exp \left(-\beta E_{\downarrow}\right)
$$

In what follows we don't specify the value of $E_{\downarrow}$, which depends on the particular details of liquid/solid system in question. We merely note that it is dependent on both the liquidliquid and liquid-solid interactions and is determined by two different factors. The positive contribution to $E_{\downarrow}$ is due to the presence of attractive interactions between the fluid particles and the atoms of the solid - a jump of a fluid particle downwards to the solid interface decreases energy. The second, negative factor stems from the presence of cohesive liquidliquid interactions; it equals the energy loss due to breaking cohesive bonds with several fluid molecules, since for the particles being in the bulk phase the number of neighbors is greater than that for particles directly on the solid. In case of chain molecules, this factor includes the contribution associated with the work required to detach the macromolecule from the melt of intertwined polymers.

- Dynamics of the film particles on the solid interface

We employ the conventional picture of such dynamics (see e.g. [37.38] and references therein) and view the motion of particles as an activated random hopping transport, constrained by hard-core interactions, between the local minima of a wafer-like array of potential wells (wavy line in Fig.5). The reason why such wells occur may be twofold: On one hand, the 
film's particles move on the solid interface and thus experience the ordering potential of solid atoms. On the other hand, such wells arise because of the mutual, collective interactions of particles in the film (as for the motion in bulk liquids). Without going into details of particle-particle and particle-substrate interactions, we suppose that for the transition to one of neighboring potential wells a particle has to overcome a potential barrier. This barrier does not create a preferential hopping direction, but results in a finite time interval $\tau$ between the consecutive hops, defined through the Arrhenius formula. The interwell distance (denoted as a) may be related either to the radius of the repulsive part of the particle-particle interactions or to the spacing between the atoms of the substrate.

We assume that all particles (except the particles at the edge of the film, which are in immediate contact with the "liquid-vapour" interface; we will call these - the "boundary particles") have symmetric transition rates: for these a probability of hop in any of four directions is $1 / 4$. Then, the diffusion coefficient of particles on solid is defined through the parameters $a$ and $\tau$ as $D=a^{2} / 4 \tau$, where both $a$ and $\tau$ depend on the form of liquid-liquid and liquid-solid interactions. Otherwise stated, $D=k T / \gamma$, where $k$ and $T$ are the Boltzmann constant and the temperature respectively, while $\gamma$ is the effective friction coefficient for motion in a liquid monolayer on the solid interface. Hard-core interactions constrain the particle hopping motion; no two particles can simultaneously occupy the same well. Thus a hop onto a well, already occupied by another fluid particle is forbidden.

- Dynamics of the boundary particles

Now we define dynamics of the boundary particles (BP), which will in some aspects be different from that of particles in the film. First, for the BP the hops in the direction to the MLE are constrained by the hard-core interactions, while hops away from the MLE are always unconstrained. Second, the boundary particles move in the vicinity of an effective "liquid-vapour" interface which influences their motion exerting a constant "restoring" force directed towards the MLE. We thus stipulate that for the BP the hops which increase the distance from the MLE occur with smaller probability $(p)$ than hops which decrease this 
distance (such hops occur with the probability $q, p<q$ ). The values of $q$ and $p$ are constant in time and are independent of the radius of the film.

Adopting the SOSM approximation [31 33], we may readily establish the relation between the magnitude of this "restoring" force (and, consequently, between the ratio $p / q$ ), and the surface tension of the interface. In this approximation, the energetic "cost" $F\left(R(t), h_{1}, h_{2}, \ldots, h_{N}\right)$ of a particular configuration of an interface (discretized as shown in Fig.5) with a fixed set of the layers radii, $R(t)$ and $\left\{h_{j}\right\}$, is given by

$$
F\left(R(t), h_{2}, h_{3}, \ldots, h_{N}\right)=P\left(\left|R(t)-h_{1}\right|\right)+\sum_{j=1}^{N-1} P\left(\left|h_{j+1}-h_{j}\right|\right)
$$

where $P(x)$ describes the interaction energy between the neighboring layers; $P(x) \approx J_{1} x^{2}$ for sufficiently small $x$ (elastic interface) and $P(x) \approx J|x|$ for $x$ being large [31] 33]. The parameter $J$ is proportional to the surface tension. Suppose next the situation as depicted in Fig.5, in which a single monolayer of length $R(t)$ appears, such that $R(t) \gg h_{1} ; h_{1} \approx h_{j} \approx$ $R_{m l e}$. Then, it follows from Eq.(3) that the cost of surface energy for having a precursor film of radius $R(t)$ is simply

$$
F\left(R(t), h_{2}, h_{3}, \ldots, h_{N}\right) \approx J R(t)
$$

i.e. it increases linearly with $R(t)$. This means, in turn, that the interface exerts a constant, independent of the film radius, pressure on the film directed towards the MLE. In other words, the BP experience an action of a constant force $f, f=-\partial F / \partial R(t)=-J$, which favors its hops in the direction to the MLE. Let us stress that in this picture only the boundary particle is subject to a surface-induced force; all other particles in the film don't "feel" the presence of the interface and thus have symmetric transition rates.

Let us discuss now the physical meaning of the "surface tension" parameter $J$. The microscopic origin of the asymmetric hopping rates stems from the mutual interactions between the particles in the film. Typical interactions in real systems are characterized by a harsh repulsion of a hard-core type at short scales and attraction at longer distances. Now, the hops of the BP away from the MLE and the hops in the direction of the MLE do not 
change the number of particles at a given $Y$ but result in stretching or shrinking of the film. Thus the change in the length of film comprising a fixed number of particles results in the change of energy. Stretching of the film will lead to an increase of energy. Conversely, shrinking of the film decreases the interparticle distances and thus results in a decrease of energy. In other words, the presence of the particle-particle attraction results in correlations between the local transition rates and spatial distribution of particles - - these tend to move towards the spatial regions in which the particle density is high. Since the density is maximal at the MLE and decreases with an increase of distance, the particles in the film experience, on average, an action of a force which is directed to the MLE. In our model this circumstance is taken into account in a mean-field fashion by introducing an integral (over all particles of the film) force which acts on the BP only and which is equivalent to the presence of a SOSM-type interface with some effective surface energy. To avoid confusion with the surface tension $J$, which appears in Eqs.(3) and (4), we will denote this effective surface energy as $W_{\leftarrow}$. In view of previous discussion, $W_{\leftarrow}$, which is the difference of the energies gained and lost due to the hop of the BP away from and in the direction to the MLE, will be defined as the work required to transport a vacancy from the edge of the film to the MLE. In contrast to the parameter $E_{\downarrow}$, the effective surface energy $W_{\leftarrow}$ is thus dependent only on the liquidliquid interactions. Using detailed balance arguments we get the following relation between $p, q$ and $W_{\leftarrow}$,

$$
\frac{p}{q}=\exp \left(-\beta W_{\leftarrow}\right)
$$

We note, finally, that by definition $W_{\leftarrow}$ equals the difference of the potential energy of vacancy placed at the MLE and the potential energy of vacancy at the edge of the film, and hence is independent of the radius and the mass of the film, provided that $R(t) \gg a$.

\section{B. Basic equations.}

We turn now to the mathematical description of the film growth focusing first on the case of capillary rise geometries. 
To specify positions of the wells, we introduce a pair of perpendicular coordinate axis, in which the $Y$-axis will be parallel to the MLE, i.e. will define the horizontal position of a given well, while the $X$-axis will measure the altitude of a given well above the MLE. Here $R(t)$ gives the height of the film relative to the origin; $R_{m l e}$ (which is a horizontal straight line) separates the film and the macroscopic meniscus and the difference $R(t)-R_{m l e}$ defines the linear extension of the film above the macroscopic meniscus. Further on, we define the variable $\eta(X, Y, t)$ - the time-dependent occupation variable of the well with coordinates $X$ and $Y$. This variable may assume two possible values; it equals 1 if the well is occupied and 0 if the well is empty.

We note now that the $X$ and the $Y$-dependences of $\eta(X, Y, t)$ are distinctly different. Along the $X$-axis we have a reservoir of particles, which maintains fixed occupation of all wells with $X=R_{m l e}$, and well-defined constant "restoring" force acting on the BPs. Consequently, we may expect that the $X$-dependence of $\eta(X, Y, t)$ is regular. In contrast, the $Y$-dependence may stem only out of fluctuation effects; the uniform boundary at the MLE insures that there is no regular dependence on the $Y$ coordinate and only the particle dynamics may cause fluctuations in $\eta(X, Y, t)$ along the $Y$-axis. In our present analysis we will disregard these fluctuations and suppose that the occupation variable varies along the $X$-axis only, i.e. $\eta(X, Y, t)=\eta(X, t)$, and the film front is a horizontal straight line. We stress that an assumption of such a type is quite consistent with experimental observations 9 [11, which show that for sufficiently smooth substrates and liquids with low volatility the width of the film front is very narrow. We also remark that regular $Y$-dependence may arise in the situation under study, if one applies, for instance, an upwardly directed temperature gradient along the $X$-axis [39]. In this case of a "forced spreading", the film's front undergoes a fingering instability resulting in a nearly periodical $Y$-dependence [39. Appearance of such effects, in general, requires very special conditions 4,39 , which are assumed to be absent.

In neglect of the fluctuations along the $Y$-axis we are faced to consider an effectively onedimensional problem in which the presence of the $Y$-direction will be accounted only through the particles' dynamics. Then, $\eta(X, t)$ can be viewed as a local time-dependent variable 
describing occupation of the site $X$ in a stochastic process in which hard-core particles perform hopping motion (with the time interval $\tau$ between the consecutive hops) on a onedimensional lattice of spacing a (see Fig.5). All particles, except the BP, have probabilities 1/4 for hops from $X$ to $X \pm a$, and probability $1 / 2$ to stay at $X$ (arising from the motion along the $Y$-axis). The BP, being at $X$, may jump to $X+a$ with probability $p$ and to $X-a$ with probability $q$, provided that this site is vacant; and may remain at $X$ with probability $1 / 2$. Further on, a source at $X=R_{m l e}$ maintains a fixed occupation of this site. This process is a generalization of a "directed walk in a lattice gas" model, studied analytically and numerically in [40,41], and here we will extend the previously elaborated continuous-space and time mean-field-type description to the more complicated process under study. In this description we will focus on the evolution of the BP mean displacement, which we denote as $R(t)$, and mean occupation (or density) of the site $X$ at time $t, \rho(X, t)=<\eta(X, t)>$, where brackets denote averages with respect to different realizations of the stochastic process.

We start with the description of the dynamics of the boundary particle, whose mean displacement is found to obey the following exact equation

$$
\tau \frac{d R(t)}{d t}=a p-a q\left(1-\rho_{1}\right)
$$

where $\rho_{1}=\rho(X=R(t)-a, t)$, i.e. the mean occupation of the site adjacent to the position of the BP.

Turning now to the dynamics of the film particles, we note that here we have to consider separately the evolution of $\rho(X, t)$ on sites $X$ of the interval $[0, R(t)-2 a]$ and at $X=R(t)-a$. Particles which may be present at the first interval are all identical. In contrast, evolution of $\rho(X, t)$ at $X=R(t)-a$ is affected by the $\mathrm{BP}$ with its asymmetric transition rates. For the first interval any (forbidden) attempt of any particle to hop onto the well already occupied by another particle is quite equivalent to the event when both simply interchange their positions, which means that hard-core exclusion is not very important for the evolution of $\rho(X, t)$ on $[0, R(t)-2 a]$. Thus, as a reasonably good approximation we suppose that on this interval the density $\rho(X, t)$ obeys a standard diffusion equation 


$$
\frac{\partial \rho(X, t)}{\partial t}=D \frac{\partial^{2} \rho(X, t)}{\partial X^{2}} ; D=\frac{a^{2}}{4 \tau},
$$

while for the dynamics of $\rho(X, t)$ at $X=R(t)-a$ we will have

$$
a \frac{d \rho_{1}}{d t}=-\left.D \frac{\partial \rho(X, t)}{\partial X}\right|_{X=R(t)-a}--\rho_{1} \frac{d R(t)}{d t},
$$

in which the first term on the right-hand-side accounts for the exchanges of identic particles between the sites $R(t)-2 a$ and $R(t)-a$; the second term describes the change in the mean occupation of the site $X=R(t)-a$ due to the motion of the BP. Here, the multiplier $d R(t) / d t$ determines the rate at which the site adjacent to the $\mathrm{BP}$ becomes vacant due to the motion of the BP. In turn, the factor $\rho_{1}$, which is the mean occupation of the site adjacent to the boundary particle, accounts in a mean-field manner for the following circumstance: Suppose that at time $t$ the $\mathrm{BP}$ is at $R(t)$ and the site $X=R(t)-a$ is vacant, i.e. $\eta(X=R(t)-a, t)=0$. Then, if at the time moment $t+\tau$ the BP makes a hop away from the MLE, it "creates" a vacancy at the previously occupied site and thus $\eta(X=R(t)-a, t+\tau)=0$ still equals zero. Therefore, the occupation of this site is not effectively changed in the case when prior to the BP hop the lhs adjacent site was vacant. Conversely, if at time $t$ the lhs adjacent to the $\mathrm{BP}$ site is occupied, i.e. $\eta(X=R(t)-a, t)=1$, and the BP hops away of the MLE, one has that $\eta(X=R(t)-a, t+\tau)=0$, i.e. is changed from one to zero.

Eqs.(6) to (8) constitute a complete, coupled system of dynamical equations describing the time evolution of the particle density $\rho(X, t)$ and the mean displacement $R(t)$ of the boundary particle (extension of the film) in capillary rise geometries.

Consider now the appropriate extension of these equations for the case of circular drops deposited on a horizontal substrate. In this case we will proceed essentially along the same lines, as in the case of capillary rise geometries. First, we will assume that the MLE and the edge of the film are ideal circular lines of radii $R_{m l e}$ and $R(t)$ respectively; the fluctuations of the film edge are disregarded. Further on, to specify the spatial positions of the wells we introduce polar coordinates $(X, \phi)$, where $X$ is the radial coordinate and $\phi$ denotes the polar angle. Supposing next that density profiles are radially symmetric, we will neglect the 
angular dependence of the density profiles. Turning now to the mathematical description of the problem, we notice that under such assumptions only Eq.(7) will be modified, while Eqs.(6) and (8) will remain the same, provided that $X$ denotes now the radial variable. Explicitly, Eq.(8) will have the form

$$
\frac{\partial \rho(X, t)}{\partial t}=D\left[\frac{\partial^{2}}{\partial X^{2}}+\frac{1}{X} \frac{\partial}{\partial X}\right] \rho(X, t)
$$

i.e. the laplacian operator will be two-dimensional, in contrast to the effectively onedimensional diffusion operator which appears in the capillary rise geometries. Eq.(9) is

to be solved subject to the boundary condition at the MLE, $\rho\left(X=R_{m l e}, t\right)=\rho_{0}$, and Eq.(8). We note that, in fact, $R_{m l e}$ is a slowly varying function of time (see Sec.1); its time dependence, however, is uncomparably weaker than an expected $\sqrt{t}$-law for growth of the film radius (see Fig.4). Thus in the following we will assume that $R_{m l e}$ is constant.

\section{Solutions of dynamical equations in case of capillary rise geometries.}

We will base our approach to the solution of coupled nonlinear Eqs.(6) to (8) on a priori assumption that $R(t)$ actually grows in time as $\sqrt{t}$ and that the density profile $\rho(X, t)$ attains a stationary form in terms of a scaled variable $\omega, \omega=\left(X-R_{m l e}\right) /\left(R(t)+a-R_{m l e}\right)$. We note that, of course, the solution so obtained must be tested for consistency with the initial assumption. Consequently, such an approach will be self-consistent if we succeed to show that there exists a finite, constant prefactor in the dependence $R(t) \sim \sqrt{t}$, for which Eqs.(6) to (8) are compatible.

Rewriting Eq.(7) in terms of the defined above scaled variable $\omega$ we have

$$
D \frac{d^{2} \rho(\omega)}{d \omega^{2}}+\omega\left(R(t)-R_{m l e}\right) \frac{d R(t)}{d t} \frac{d \rho(\omega)}{d \omega}=0
$$

Let us denote

$$
A_{m}=\frac{1}{2 D} \frac{d\left(R(t)-R_{m l e}\right)^{2}}{d t}
$$


In view of our assumption this parameter is expected to be a time-independent constant, which (when found explicitly) will define the growth law of $R(t)$.

To find $A_{m}$ we will proceed as follows: We notice first that since $d R(t) / d t \rightarrow 0$ when $t \rightarrow \infty$, Eq.(6) insures that $\rho_{1}$ rapidly, at rate $\left|d \rho_{1} / d t\right| \ll d R(t) / d t$, approaches a constant value $\tilde{\rho}_{1}, \tilde{\rho}_{1}=1-p / q$. Then, solving the differential Eq.(10) subject to the boundary conditions $\rho(\omega=0)=\rho_{0}$ and $\rho(\omega=1)=\tilde{\rho}_{1}$ we find

$$
\rho(\omega)=\rho_{0}+\left(\tilde{\rho}_{1}-\rho_{0}\right) \frac{\operatorname{erf}\left(\omega \sqrt{A_{m} / 2}\right)}{\operatorname{erf}\left(\sqrt{A_{m} / 2}\right)},
$$

where $\operatorname{erf}(x)$ denotes the error function. From Eq.(12) we then obtain

$$
\left.\frac{d \rho(\omega)}{d \omega}\right|_{\omega=1}=-\sqrt{\frac{2 A_{m}}{\pi}} \frac{\left(\rho_{0}-\tilde{\rho}_{1}\right) \exp \left(-A_{m} / 2\right)}{\operatorname{erf}\left(\sqrt{A_{m} / 2}\right)}
$$

On the other hand, rewriting Eq.(8) in terms of the scaled variable $\omega$ and neglecting transient terms, we will get

$$
\left.\frac{d \rho(\omega)}{d \omega}\right|_{\omega=1}=-A_{m} \tilde{\rho}_{1}
$$

On comparing the rhs of Eqs.(13) and (14) we arrive at a closed with respect to $A_{m}$ equation, which defines its dependence on the given parameters $E_{\downarrow}$ and $W_{\leftarrow}$,

$$
\sqrt{\frac{\pi A_{m}}{2}} \exp \left(\frac{A_{m}}{2}\right) \operatorname{erf}\left(\sqrt{\frac{A_{m}}{2}}\right)=\frac{1-\exp (-\beta s)}{\exp \left(\beta W_{\leftarrow}\right)-1}
$$

in which $s$ denotes the difference

$$
s=E_{\downarrow}-W_{\leftarrow}
$$

A simple analysis shows that whenever the rhs of Eq.(15) is positive, Eq.(15) has a single positive solution and thus $A_{m}$ is actually a well-defined positive constant. Consequently, we may claim that the mean displacement of the boundary particle (or, in other words, the mean extension of the wetting film) obeys

$$
R(t)=R_{m l e}+\sqrt{2 A_{m} D t}
$$


i.e. the form of Eq.(1) in which the "fitting" parameter $\alpha$ (Sec.II) is equal to the product of the diffusion coefficient and the parameter $A_{m}$. Eq.(17) is the primary analytical result of our analysis and agrees with the experimentally observed time dependence [9, [1, 16, [17].

Now, we estimate analytically the dependence of $A_{m}$ on the pertinent parameters in the asymptotic limit when $A_{m}$ is small or large. It follows from Eq.(15) that $A_{m}$ is small when the rhs of Eq.(15) is small. It happens, namely, when either the inequality $\beta E_{\downarrow}>\beta W_{\leftarrow} \gg 1$ holds (what may be thought off as the case of liquids with high cohesion energy and strong attraction to the substrate), or when the parameter $s$ is sufficiently small, such that $\beta s \ll$ $\exp \left(\beta W_{\leftarrow}\right)-1$. When either of these inequalities is fulfilled $A_{m}$ is given explicitly by

$$
A_{m} \approx \frac{(1--\exp (-\beta s))}{\left(\exp \left(\beta W_{\leftarrow}\right)-1\right)}
$$

We note now that growth of the film occurs as long as the parameter $s$, Eq.(16), is positive, i.e. as long as $E_{\downarrow}$ exceeds the work $W_{\leftarrow}$ needed to transport a vacancy from the edge of the film to the macroscopic liquid edge. Therefore, the parameter $s$ is the key property which distinguishes whether the monolayer will grow or not; thus it seems natural to define $s$ as the microscopic analogue of the spreading parameter $S$ - the property which rules spreading of liquids at the macroscopic scales. Consequently, we will call $s$ the microscopic spreading parameter.

Now, $A_{m}$ may be large when the rhs of Eq.(15) is large, which happens when $\beta W_{\leftarrow} \ll 1$ and $s$ is sufficiently large. In this case $A_{m}$ reads

$$
A_{m} \approx-2 \ln \left(\beta W_{\leftarrow}\right)
$$

Behavior as in Eq.(19) may be realized experimentally in case of liquids with low cohesion energy, which are volatile in two-dimensions, but not volatile in 3D. An example of such a liquid is squalane (see [10] for details).

It may be worthwhile to remark that the behavior of the density profiles defined by Eq.(12) is very different in the limits when $A_{m}$ is small or large. When $A_{m}$ is small $\rho(\omega, t)$ displays a linear dependence on $\omega$ (and thus on $X), \rho(\omega, t) \approx \rho_{0}+\left(\tilde{\rho}_{1}-\rho_{0}\right) \omega$, while for 
the situations in which $A_{m}$ is large it shows much stronger variation with $\omega$. In neither case, however, one can assume the liquid monolayer in capillary rise geometries to be an incompressible fluid.

Consider now the time evolution of the mass $M(t)$ of the film, defined as

$$
M(t) \approx \int_{R_{m l e}}^{R(t)} d X \rho(X, t)
$$

Changing the variable of integration and making use of Eq.(12) we get

$$
\begin{gathered}
M(t) \approx\left(R(t)-R_{m l e}\right) \int_{0}^{1} d \omega \rho(\omega)= \\
=\left(R(t)-R_{m l e}\right) \exp \left(A_{m} / 2\right)\left(1--\exp \left(-\beta W_{\leftarrow}\right)\right),
\end{gathered}
$$

which shows that $M(t)$ also grows in proportion to $\sqrt{t}$, in accord with experimental observations [9,14]. Eqs.(17) and (21) thus imply that mean density of particles in the film, $\bar{\rho}$, remains constant,

$$
\bar{\rho}=\frac{M(t)}{\left(R(t)-R_{m l e}\right)}=\exp \left(A_{m} / 2\right)\left(1--\exp \left(-\beta W_{\leftarrow}\right)\right)
$$

In case of small $A_{m}$ the mean density is close to unity, while for progressively large $A_{m}$ it tends to zero. This behavior is illustrated in Fig.6, where we plot the functions $M(t) / \sqrt{t}$, $\left(R(t)-R_{m l e}\right) / \sqrt{t}$ and $\bar{\rho}$ versus the transition probability $q, q=1 /\left(1+\exp \left(-\beta W_{\leftarrow}\right)\right)$.

We close this subsection with some comments concerning spreading kinetics in situations in which $W_{\leftarrow}=0$, i.e. when "liquid-vapour" interface is absent. This case is somewhat peculiar, since the rhs of Eq.(15) diverges, which means that $A_{m}$ is no longer a well-defined constant but rather is some increasing function of time. Eq.(19) shows that $A_{m}$ diverges logarithmically when $W_{\leftarrow}$ tends to zero; thus one may expect that $A_{m}$ grows in proportion to $\ln (t)$ when $W_{\leftarrow}$ is exactly equal to zero. In [36] we have shown that it is actually so and $A_{m}$ displays a slow logarithmic growth

$$
A_{m} \approx \ln \left(\frac{4 \rho_{0}^{2} D t}{\pi a^{2}}\right)
$$


at sufficiently large times. This means, in fact, that the presence (or absence) of attractive liquid-liquid interactions (or of the SOSM interface in our description) does not affect significantly the spreading kinetics resulting only in slowly varying in time prefactors.

\section{Solutions of dynamical equations for circular droplets on a horizontal substrate}

Let us now examine the growth law of the precursor film for circular droplets. In this case the density profiles, defined by Eq.(9), do not attain the stationary form $\rho(\omega)$; the gradient term $X^{-1} \partial / \partial X$ in the laplacian operator does not allow to represent the complete time dependence of $\rho(X, t)$ in terms of the scaled variable $\omega$ only. We thus shall seek for the solution of Eq.(9) of the form $\rho(X, t)=\rho(\omega, t)$. For this, Eq.(9) reads

$$
\frac{R_{m l e}^{2}}{D \mu^{2}(t)} \frac{\partial \rho(\omega, t)}{\partial t}=\frac{\partial^{2} \rho(\omega, t)}{\partial \omega^{2}}+\left(\frac{1}{\omega+\mu(t)}+A_{m} \omega\right) \frac{\partial \rho(\omega, t)}{\partial \omega},
$$

in which we have denoted $\mu(t)=R_{m l e} /\left(R(t)-a-R_{m l e}\right)$. The solution of Eq.(24) can be found recursively, expanding $\rho(\omega, t)$ in the inverse powers of the diffusion coefficient

$$
\rho(\omega, t)=\sum_{n=0}^{\infty} D^{-n} \Psi_{n}(\omega, t)
$$

In doing so, we will obtain for the zeroth term

$$
\begin{aligned}
\Psi_{0}(\omega, t)= & \rho_{0}+\left(\tilde{\rho}_{1}-\rho_{0}\right) \int_{0}^{\omega} \frac{d \omega}{\omega+\mu(t)} \exp \left(-\omega^{2} A_{m} / 2\right) \times \\
& \times\left\{\int_{0}^{1} \frac{d \omega}{\omega+\mu(t)} \exp \left(-\omega^{2} A_{m} / 2\right)\right\}^{-1},
\end{aligned}
$$

while higher-order terms will be defined through

$$
\frac{\partial^{2} \Psi_{n}(\omega, t)}{\partial \omega^{2}}+\left(\frac{1}{\omega+\mu(t)}+A_{m} \omega\right) \frac{\partial \Psi_{n}(\omega, t)}{\partial \omega}=\frac{R_{m l e}^{2}}{\mu^{2}(t)} \frac{\partial \Psi_{n-1}(\omega, t)}{\partial t}
$$

Straightforward, but rather tedious analysis which will be presented elsewhere [42], shows however that only the zeroth term is relevant; the higher-order $\Psi_{n}(\omega, t)$ define only small at any $t$ and $\omega$ corrections and $\rho(\omega, t) \approx \Psi_{0}(\omega, t)$ occurs to be quite an accurate approximation. 
Consider now behavior of $\Psi_{0}(\omega, t)$ defined by Eq.(26). At relatively short times, when $R(t)$ does not exceed significantly $R_{m l e}$, the function $\mu(t) \gg 1$. Consequently, in this time regime we may safely neglect the variable $\omega(0 \leq \omega \leq 1)$ compared to $\mu(t)$ in the function $(\omega+\mu(t))^{-1}$. Then, Eq.(26) simplifies to the form of Eq.(12), which defines the stationary density profiles in capillary rise geometries. This means, in turn, that at early stages of the film growth its radius obeys exactly the law in Eq.(17) with the parameter $A_{m}$ defined by Eq.(15). Of course, this result is not unexpected on physical grounds - when $R(t)$ is comparable to $R_{m l e}$ effects of curvature can not be important. Turning next to the opposite limit, when $R(t) \gg R_{m l e}$ and thus $\mu(t) \ll 1$, we find from Eq.(26),

$$
\left.\frac{d \rho(\omega, t)}{d \omega}\right|_{\omega=1} \approx-\left(\rho_{0}-\tilde{\rho}_{1}\right) \exp \left(-A_{m} / 2\right)\left\{\int_{0}^{1} \frac{d \omega}{\omega+\mu(t)} \exp \left(-\omega^{2} A_{m} / 2\right)\right\}^{-1}
$$

Comparing Eqs.(28) and (14) we will obtain for the parameter $A_{m}$ :

$$
\frac{1-\exp (-\beta s)}{\exp \left(\beta W_{\leftarrow}\right)-1} \approx \exp \left(A_{m} / 2\right) A_{m} \int_{0}^{1} \frac{d \omega}{\omega+\mu(t)} \exp \left(-\omega^{2} A_{m} / 2\right)
$$

Now, since $\mu(t) \rightarrow 0$ when $t$ progresses, the value of the integral in Eq.(29) is dominated by the lower limit, i.e. vicinity of $\omega=0$. Neglecting then the $\operatorname{exponent} \exp \left(-\omega^{2} A_{m} / 2\right)$, which will contribute only to the second order in powers of $A_{m}$, and integrating, we get the following relation

$$
\frac{1-\exp (-\beta s)}{\exp \left(\beta W_{\leftarrow}\right)-1} \approx A_{m} \ln \left(\frac{1}{\mu(t)}\right)
$$

which yields for $t \gg R_{m l e}^{2} / 2 D,\left(R(t) \gg R_{m l e}\right)$,

$$
\begin{gathered}
A_{m} \approx \frac{1-\exp (-\beta s)}{\exp \left(\beta W_{\leftarrow}\right)-1} \frac{2}{\ln \left(2 D t / R_{m l e}^{2}\right)}\{1+ \\
\left.+\frac{\ln \left[\ln \left(2 D t / R_{m l e}^{2}\right)+\left(\exp \left(\beta W_{\leftarrow}\right)-1\right) / 2(1-\exp (-\beta s))\right]}{\ln \left(2 D t / R_{m l e}^{2}\right)}+\ldots\right\}
\end{gathered}
$$

Eq.(31) thus shows that in this time limit the radius of the monolayer grows as $R(t) \sim$ $\sqrt{t / \ln (t)}$, in accord with the prediction of de Gennes and Cazabat 29. The prefactors in the growth law in Eq.(31) are, however, different from these obtained in [29]. The density profiles corresponding to this time regime attain the form 


$$
\rho(X, t) \approx \rho_{0}+\left(\tilde{\rho}_{1}-\rho_{0}\right) \frac{\ln \left(X / R_{m l e}\right)}{\ln \left(R(t) / R_{m l e}\right)}
$$

which shows a logarithmically slow variation with $X$. This means apparently that in the regime $R(t) \gg R_{m l e}$ the liquid monolayer arising during spreading of a circular droplet can be approximately viewed as an incompressible 2D fluid.

Finally, let us estimate the time evolution of the mass of particles in the film and of the mean density. For the mass we obtain

$$
M(t)=2 \pi \int_{R_{m l e}}^{R(t)} X d X \rho(X, t)
$$

where $\rho(X, t)$ is defined in the large- $t$ limit by Eq.(32). Substituting Eq.(32) into the Eq.(33) and integrating, we get

$$
M(t) \approx \pi\left[\left(R^{2}(t)-R_{m l e}^{2}\right)\left(\rho_{0}+\frac{\rho_{0}-\tilde{\rho}_{1}}{2 \ln \left(R(t) / R_{m l e}\right)}+R^{2}(t)\left(\tilde{\rho}_{1}-\rho_{0}\right)\right]\right.
$$

Consequently, the mean density of particles in the monolayer obeys

$$
\bar{\rho} \approx \tilde{\rho}_{1}+\frac{\rho_{0}-\tilde{\rho}_{1}}{2 \ln \left(R(t) / R_{m l e}\right)}
$$

which means that in situations, in which the SOSM interface enclosing the hard-sphere fluid is present, the mean density of particles in the film tends to a constant value, dependent on the surface tension of the interface. In the absence of the interface (i.e. when $W_{\leftarrow}=0$ ), the mean density decreases in time in proportion to $\rho_{0} / \ln (t)$.

\section{SUMMARY AND DISCUSSION}

To conclude, we have presented here both experimental and theoretical analysis of kinetics of thin liquid films spreading on solid interfaces.

In the experimental part we have described results of recent ellipsometric measurements

of the time dependent thickness profiles and of the growth rate of the first layer on top of interface. Experiments, which were carried out on different types of bare or grafted solid substrates and were performed with two different types of liquids - melts of light 
polymers (PDMS) and liquid of spherical rigid molecules (TK), have shown the extraction of a molecularly thin precursor film, advancing well ahead of the macroscopic liquid edge, and also displayed several other features observed in earlier works [9]11. Namely, the appearance of stepped, "terraced" shapes of the liquid droplet at the microscopic, molecular scales in case of the PDMS droplets and also, for both types of liquids, confirmed that the first layer on top of solid grows in proportion to $\sqrt{t}$.

We have proposed an analytical model in which the spreading monolayer is viewed as a hard-sphere fluid enclosed by an effective "liquid-vapour" interface which stabilizes fluid and mimics, in a mean-field fashion, the presence of cohesive liquid-liquid interactions. The macroscopic drop in our description is considered as a reservoir of particles of an infinite capacity, which feeds the film. The model allows an analytical solution; we have found explicit expressions describing the growth of the first layer for both the case of capillary rise geometries and circular droplets on a horizontal solid, as well as determined the time evolution of the mass of particles in the film, the mean density and dynamical density profiles. For the case of capillary rise geometries we have shown that $R(t)$ (the extension of the film above the macroscopic meniscus) grows in time as $\sqrt{2 A_{m} D t}$, where $D$ is the "bare" diffusion coefficient describing random motion of a particle on solid interface, $A_{m}$ is the parameter which is dependent on the magnitude of liquid-liquid and liquid-solid interactions. This parameter is determined implicitly, in form of a transcendental equation; in limiting situations its explicit forms are found. Mass of particles in the film is also shown to grow in proportion to $\sqrt{t}$, which means that the mean density stays constant. We have also shown that local density of particles in the film varies essentially with the altitude above the meniscus. Now, in case of circular droplets on a horizontal substrate, our analytical findings are as follows: we predict that at short times, when the radius of the first layer is comparable to the radius of the macroscopic liquid edge, the growth of the film occurs essentially like in the capillary rise geometries. Within the opposite limit, when $R(t)$ exceeds substantially $R_{m l e}$, we have established that the parameter $A_{m}$ decreases in time as $1 / \ln (t)$, which yields 
$R(t) \sim \sqrt{t / \ln (t)}$, in accord with the theory of de Gennes and Cazabat [29]. We have also shown that in this time regime the density profile is described by a logarithmically slow function of the radial distance from the macroscopic liquid edge, i.e. an assumption that the monolayer can be viewed as an incompressible 2D fluid [29] may be physically plausible when $R(t) \gg R_{m l e}$. Explicit results for the mass of particles and for the mean density are also presented.

Further on, our analytical results suggest that the physical mechanism responsible for the $\sqrt{t}$-law for growth of monolayers is associated with the diffusive transport of "vacancies" from the edge of the film to the macroscopic liquid edge. Arriving to the MLE a vacancy perturbes the equilibrium between the film and the bulk liquid; and then is filled by a fluid particle from the reservoir. We specified the microscopic spreading parameter " $s$ ", which distinguishes whether the monolayer will grow or not. In our picture, this parameter is equal to the difference of the energy gained by filling a vacancy at the macroscopic liquid edge by a fluid particle and the work required to transport a vacancy from the edge of the film to the MLE. Growth of a monolayer does not take place if this parameter is negative. In view of this we may comment that it is somewhat misleading to call the $\sqrt{t}$ growth of monolayers as "diffusive"; here it describes the growth of the mean displacement, i.e. spreading, which is exactly zero for diffusive-type processes. Spreading of liquid monolayers is rather reminiscent, in view of the physics involved, of the phenomena of directional solidification or of melting, in which the spreading of a "new" phase front is controlled by the rate at which the particles of an "old" phase diffuse away of it [43].

Finally, we remark that the model discussed here has several evident shortcomings and is to be improved in several directions. First, assuming the reservoir to have an infinite capacity, we certainly limit our model description to only some intermediate time regime, which is, of course, quite extended in time but nonetheless does not cover all stages of the liquid droplet spreading. The third point of our model, which is some sort of a meanfield type assumption, may be more bothering since it substitutes the collective attractive 
interaction of all particles in the film by some effective one, imposed on the particles at the edge of the film only. It is heuristically resolved here by taking the effective surface tension equal to the work required to transport a vacancy from the edge of the film to the macroscopic liquid edge, but surely a model involving explicitly the cohesive interactions is required. Let us discuss within the framework of the present analytical approach the final stage of spreading of a liquid droplet. In our approach, allowing the reservoir to be exhausted, i.e. violating the boundary condition in Eq.(2) at some moment of time, would yield immediate termination of the film growth; the impenetrable "liquid-vapour" interface embracing the molecules on solid interface will prevent further spreading and the film will form a stable, circular liquid patch of molecular thickness. For monolayers this is the case for 2D non-volatile liquids (PDMS with molecular mass above 2000). For 2D volatile liquids the molecular diffusion ultimately destroys such structure [9]. Experiments performed with light PDMS molecules and squalane [9] (see also [21] and references therein) clearly show that a molecularly thin liquid patch, appearing after the central part of the droplet is emptied, is not stable and continues to spread indefinitely forming a two-dimensional gas. Experimental data, described here in Fig.3, show that the film ceases to grow when the reservoir disappears and thus seemingly conforms to the prediction of our model. We remind, however, that the PDMS molecules involved in this study were rather long, containing more than one hundred monomers. Effects of the reduced diffusivity or, possibly, entanglements, may matter here and give rise to extremely slow spreading inaccessible at experimentally available time scales.

\section{Acknowledgments.}

S.F.B acknowledges the support of ONR Grant N 00014-94-1-0647 and by the University of Paris VI. G.O. acknowledges financial support from the FNRS, Belgium. 


\section{REFERENCES}

$\S \quad$ Unité de Recherche Associée au CNRS (URA 765)

• Unité de Recherche Associée au CNRS (URA 792)

[1] P.G.de Gennes, Rev. Mod. Phys. 57, 827 (1985)

[2] A.M.Cazabat, Contemp. Phys. 28, 347 (1987)

[3] P.Ball, Nature (London) 337, 624 (1989)

[4] L.Léger and J.F.Joanny, Rep. Prog. Phys. 72, 431 (1992)

[5] L.H.Tanner, J. Phys. D 12, 1478 (1979)

[6] F.Brochard, J. Chem. Phys. 84, 4664 (1986)

[7] F.Brochard-Wyart, P.G. de Gennes and H.Hervet, Adv. Colloid and Interface Sci. 34, $561(1991)$

[8] A.Carré, J.C.Gastel and M.R.E.Shanahan, Nature 379, 432 (1996)

[9] F.Heslot, N.Fraysse and A.M.Cazabat, Nature (London) 338, 640 (1989)

[10] F.Heslot, A.M.Cazabat and P.Levinson, Phys. Rev. Lett. 62, 1286 (1989)

[11] F.Heslot, A.M.Cazabat, P.Levinson and N.Fraysse, Phys. Rev. Lett. 65, 599 (1990)

[12] F.Tiberg and A.M.Cazabat, Europhys. Lett. 25, 205 (1994)

[13] S.Villette, M.P.Valignat, A.M.Cazabat, L.Jullien anf F.Tiberg, Langmuir 12, 825 (1996)

[14] M.P.Valignat, N.Fraysse, A.M.Cazabat, P.Levinson, F.Heslot and M.Cazabat, Colloids and Surfaces 83, 193 (1994)

[15] N.Fraysse, M.P.Valignat, A.M.Cazabat, F.Heslot and P.Levinson, J. of Colloid Inter. Sci. 158, 27 (1993)

[16] F.Heslot, A.M.Cazabat and N.Fraysse, J. Phys. Cond. Matter 1, 5793 (1989) 
[17] A.M.Cazabat, N.Fraysse and F.Heslot, Colloids and Surfaces 52, 1 (1991)

[18] J.De Coninck, S.Hoorelbeke, M.P.Valignat and A.M.Cazabat, Phys. Rev. E 48, 4549 (1993)

[19] J.De Coninck, U.D’Ortona, J.Koplik and J.R.Banavar, Phys. Rev. Lett. 74, 928 (1995)

[20] U.D’Ortona, J.De Coninck, J.Koplik and J.R.Banavar, Phys. Rev. E 53, 562 (1996)

[21] J.De Coninck, N.Fraysse, M.P.Valignat and A.M.Cazabat, Langmuir 9, 1906 (1993)

[22] A.Lukkarinen, D.B.Abraham, M.Karttunen and K.Kaski, Phys. Rev. E 51, 2199 (1995)

[23] K.Kaski, Europhys. News 26, 23 (1995)

[24] J.F.Joanny and P.G.de Gennes, J. Phys. (Paris) 47, 121 (1986)

[25] J.Van Alsten and S.Granick, Phys. Rev. Lett. 61, 2570 (1988)

[26] H.-W.Hu, G.A.Carson and S.Granick, Phys. Rev. Lett. 66, 2758 (1991)

[27] M.L.Gee, P.M.McGuiggan, J.N.Israelachvili and A.M.Homola, J. Chem. Phys. 93, 1895 (1990)

[28] P.A.Thompson, G.S.Grest and M.O.Robbins, Phys. Rev. Lett. 68, 3448 (1992)

[29] P.G.de Gennes and A.M.Cazabat, C. R. Acad. Sci. 310, 1601 (1990)

[30] D.B.Abraham, in Phase Transition and Critical Phenomena, eds.: C.Domb and J.L.Lebowitz (Academic Press, New York, 1986) Vol 10.

[31] D.B.Abraham, P.Collet, J.De Coninck and F.Dunlop, Phys. Rev. Lett. 65, 195 (1990)

[32] D.B.Abraham, P.Collet, J.De Coninck and F.Dunlop, J. Stat. Phys. 61, 509 (1990)

[33] J.De Coninck, F.Dunlop and F.Menu, Phys. Rev. E 47, 1820 (1993)

[34] D.Beaglehole, Physica B 100, 163 (1980); D.Beaglehole, E.Z.Radlinska, B.W.Ninham and H.Christenson, Langmuir 7, 1843 (1991) 
[35] S.F.Burlatsky, G.Oshanin, A.M.Cazabat and M.Moreau, Phys. Rev. Lett. 76, 86 (1996)

[36] S.F.Burlatsky, G.Oshanin, A.M.Cazabat, M.Moreau and W.Reinhardt, Phys. Rev. E, to appear

[37] R.Aveyard and D.A.Haydon, An Introduction to the Principles of Surface Chemistry (Cambridge University Press, Cambridge, 1973)

[38] T.D.Blake and J.M.Haynes, J. Colloid Interface Sci. 30, 421 (1969)

[39] A.M.Cazabat, F.Heslot, S.M.Troian and P.Carles, Nature 346, 824 (1990)

[40] S.F.Burlatsky, G.Oshanin, A.Mogutov and M.Moreau, Phys. Lett. A 166, 230 (1992)

[41] S.F.Burlatsky, G.Oshanin, M.Moreau and W.Reinhardt, Motion of a driven tracer particle in a one-dimensional lattice gas, submitted to Phys. Rev. E

[42] S.F.Burlatsky, A.M.Cazabat, M.Moreau and G.Oshanin, to be published

[43] J.S.Langer, Lectures in the Theory of Pattern Formation, in "Chance and Matter", eds.: J.Souletie, J.Vannimenus and R.Stora (North-Holland, Amsterdam, 1987)

\section{Figure Captions.}

Fig.1. Time evolution of the thickness profiles of a TK droplet.

Fig.2. Spreading of a TK droplet. Dynamical thickness profiles (top); Growth of the radius $R(t)$ of the first layer vrs $\sqrt{t}$ (bottom).

Fig.3. Spreading of a PDMS droplet. Dynamical thickness profiles (top); Growth of the radius $R(t)$ of the first layer vrs $\sqrt{t}$ (bottom).

Fig.4. Spreading of a PDMS droplet at high relative humidity. Large droplet (top); small droplet (bottom).

Fig.5. Schematic picture of a liquid monolayer spreading on solid interface. Wavy line depicts the effective "energy" landscape arising due to liquid-liquid and liquid-solid interactions. 
Fig.6. Plot of the analytical dependences of the functions $M(t) / \sqrt{t}$ (curve $(1)) ;(R(t)-$ $\left.R_{m l e}\right) / \sqrt{t}$ (curve $\left.(2)\right)$ and the mean density $\bar{\rho}$ (curve $\left.(3)\right)$ versus the parameter $q=1 /(1+$ $\left.\exp \left(\beta W_{\leftarrow}\right)\right)$. 


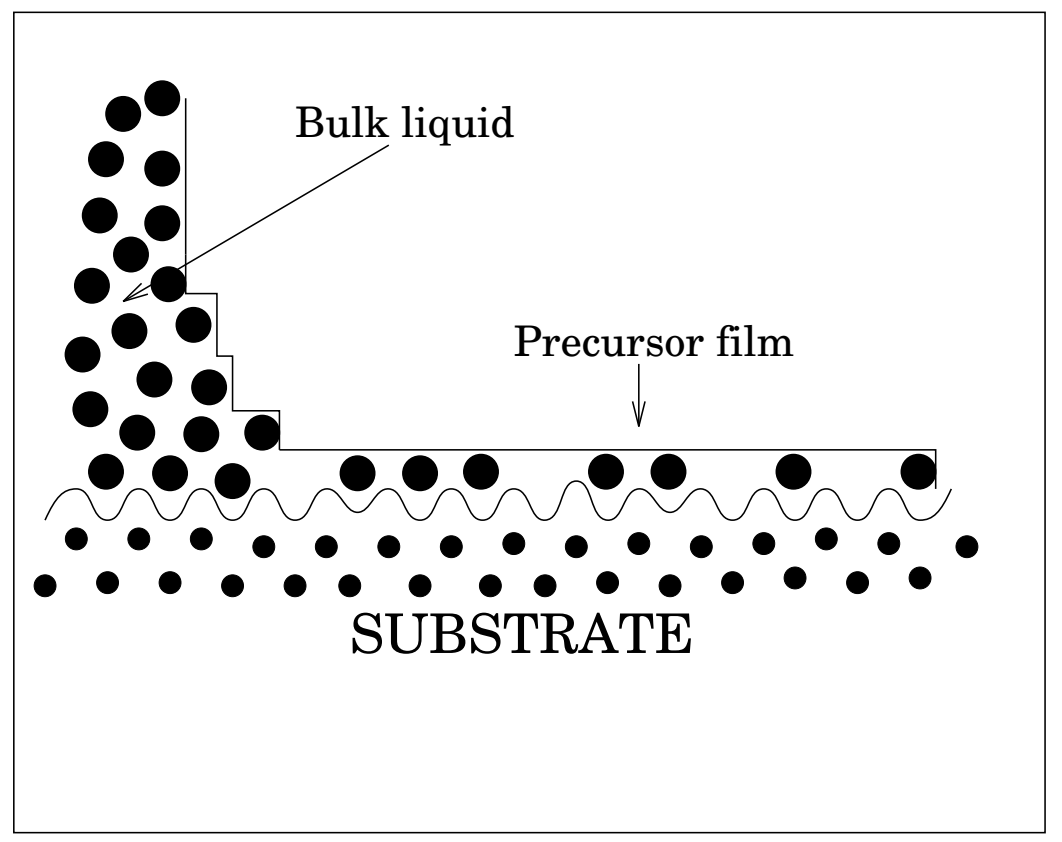


Fig.6

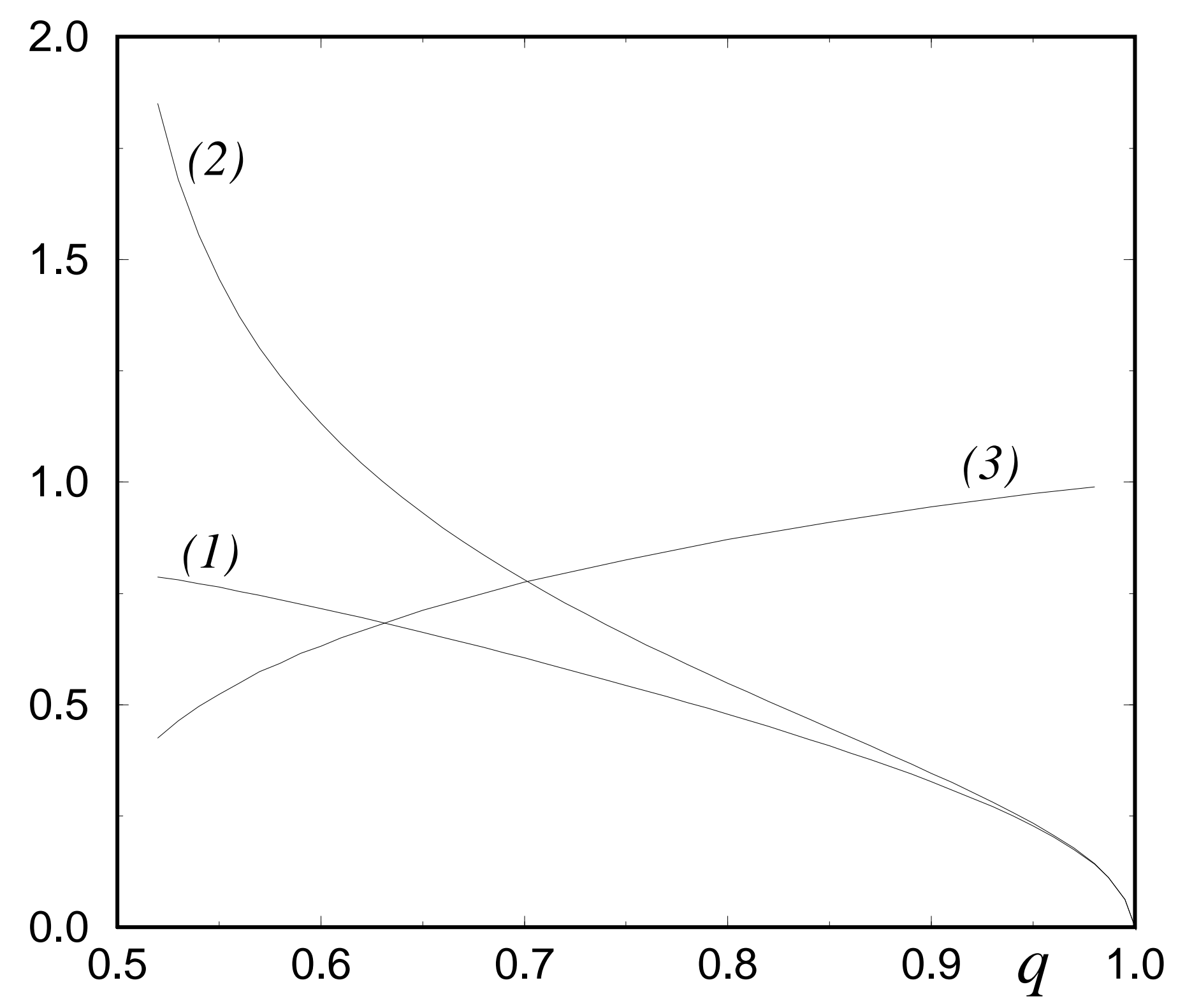

